\title{
SEMÁNTICAS FORMALES PARA EL PROCESAMIENTO DEL DISCURSO Y LA NEGACIÓN
}

\author{
Begoña Carrascal \\ Isabel Gómez Txurruka \\ Kepa Korta
}

\begin{abstract}
The main goal of this paper is twofold. On the one hand, we will present two of the most important contemporary semantic theories for discourse processing: Situation Semantics or SS (Barwise and Perry (1993)) and Discourse Representation Theory or DRT (Kamp (1981)). Both are formal theories, and therefore, they meet a necessary -though not sufficient- requirement for their eventual implementation. On the other hand, we will study a classical issue in the theory of language, namely, natural language negation. We will consider its treatment in these semantic theories, trying to show why negation has always resisted to a satisfactory treatment, and remains as an unsolved problem from Aristotle to contemporary philosophy, syntax, semantics and pragmatics (Hom (1989)). Then, we will make a (modest) proposal that will be showed to solve some of the problems of SS and DRT for treating negation. Our proposal consists in linking negation with a theory of informational partition in the frame of an enriched version of DRT called Segmented DRT (Asher (1993)).
\end{abstract}

Este trabajo tiene dos objetivos principales. Por una parte, pretendemos presentar dos de las teorías semánticas actuales más importantes en procesamiento del discurso: la semántica de situaciones (SS) y la teoría de representación del discurso (DRT). Se trata de dos semánticas formales, requisito indispensable, aunque no suficiente, para una eventual implementación. Por otra parte, abordaremos un problema clásico en teoría del lenguaje: la negación. Consideraremos su tratamiento en cada una de estas teorías y mostraremos algunos de los motivos por los que la negación se ha resistido a un tratamiento satisfactorio y ha permanecido como problema no resuelto desde Aristóteles hasta la filosofía, sintaxis, semántica y pragmática actuales. A continuación, realizaremos una propuesta (modesta) que permite superar algunas de las limitaciones de estas teorías para tratar la negación, que consiste en vincularla con el fenómeno de la partición informacional (tema/foco) en el marco de una extensión de DRT, DRT con segmentos o SDRT. 


\section{SEMÁNTICAS FORMALES}

Entre las semánticas formales que modelizan la interpretación del lenguaje natural destacan por el alto nivel de desarrollo alcanzado, SS y DRT. Surgen de modo independiente a principios de los 80 -SS en Barwise y Perry (1981), Barwise y Perry (1983); DRT en Kamp (1981)- aunque comparten varias características importantes: son teorías dinámicas, en cuanto que el significado de una proferencia es una función entre contextos; se basan en una novedosa idea de parcialidad de la información transmitida por una proferencia; ambas son semánticas realistas (aunque DRT compatibiliza el realismo de la interpretación modelística con el conceptualismo que puede desarrollarse en el nivel intermedio de la representación); finalmente, ambas son teorías semánticas atípicas en tanto que incluyen aspectos que tradicionalmente han sido considerados como pertenecientes al dominio de la pragmática.

\section{La semántica de situaciones}

Barwise y Perry (1981) presentan originalmente la semántica de situaciones como un tipo especial de semántica que supera problemas inherentes a la semántica modelística del lenguaje natural. El desarrollo de tal semántica creó la necesidad de una teoría general de la información: una teoría matemática del flujo, transmisión y almacenamiento de la información, que denominaron teoría de situaciones. A partir de aquí la semántica de situaciones se considera como una aplicación de las herramientas de la teoría de situaciones al estudio del significado, de modo que incluso puede pensarse en diversas semánticas, según el tipo de lenguaje o fragmento que se pretenda estudiar.

Introducimos, en primer lugar, algunos conceptos básicos de la teoría de situaciones necesarios para la presentación de la semántica de situaciones que luego realizaremos.

\subsection{Una teoría que sustituye los mundos por situaciones}

Las situaciones son partes del mundo. Nosotros discutiendo la redacción final de este trabajo, el partido de pelota de ayer, yo diciéndote a ti "son las diez"..., todas estas cosas son situaciones. Las situaciones son primitivos de la teoría, es decir, no se definen sobre ningún otro primitivo. Los humanos las individuan o discriminan como partes del mundo y guían su comportamiento de acuerdo con ellas.

La información está en el mundo. Fluye a través de las situaciones. Examinemos un ejemplo que ilustra esto. Piénsese en la situación que consiste en un tronco cortado. La situación lleva mucha información: la edad del árbol cuando fue cortado, el tipo de instrumento utilizado para ello, la orientación con respecto a los puntos cardinales,... En virtud de diversas relaciones legaliformes, naturales o convencionales, denominadas restricciones, entre este tipo de situación y otros tipos, el tronco cortado transporta información sobre situaciones diferentes y más o menos remotas.

La herramienta básica de la teoría de situaciones para representar unidades de información es la noción de infón. ${ }^{1}$ Un infón se compone de una relación, un conjunto de papeles

\footnotetext{
'Debemos subrayar que los infones son objetos teóricos -de la teoría- para representar unidades de información pero que no se quiere decir con eso que se consideren algo así como los objetos informacionales reales -sean lo que sean éstos- que fluyen en el mundo.
} 
argumentales para la relación, una ubicación espacio-temporal y un item de polaridad $i$ tal que $i \in\{1,0\}$. Así, el infón siguiente

(1) «RUNS, Mary, $l, 1 »$

representa la información de que María corre en la ubicación espacio-temporal $l$. Por otra parte, la información de que María no corre en $l$ se representa mediante el infón

(2) «RUNS, Mary, $l, 0 »$

Los infones (1) y (2) son recíprocamente duales. Se dice que un infón $\sigma$ es un hecho cuando alguna situación $s$ lo soporta. Se escribe $s=\sigma$ y se lee $s$ soporta a $\sigma$, o $\sigma$ es un hecho en $s$, o $\sigma$ ocurre en $s .^{2}$

De este modo, puede establecerse como principio en teoría de situaciones que si $s \mid=\sigma$ entonces $s \mid \neq \bar{\sigma}$, es decir, si una situación $s$ soporta un infón $\sigma$, entonces no soporta su infón dual $\bar{\sigma}$; en otras palabras, las situaciones, o mejor dicho, la información que una situación soporta es consistente. Ahora bien, la conversa no se cumple; no podemos sostener que si $s \mid \neq \sigma$ entonces $s \mid=\bar{\sigma}$. Si una situación no soporta un infón no hay ninguna base para afirmar que esa situación soporte su dual, lo que significa que dada una situación no podemos decidir sobre la factualidad de infones que no ocurren en dicha situación. Con esto se recoge una de las principales características de la teoría y semántica de situaciones: la parcialidad de la información.

Las situaciones y los infones son dos de los primitivos fundamentales de la teoría de situaciones junto con las relaciones, los individuos, las ubicaciones espacio-temporales, la polaridad, los parámetros, etc.

\subsection{La teoría relacional del significado}

La semántica de situaciones se ocupa del significado de las proferencias de lenguaje natural, no de las oraciones. Adopta la perspectiva austiniana que considera al lenguaje como acción. El significado oracional es un factor importante, pero sólo un factor, para determinar el significado de la proferencia realizada por un agente que se dirige a otro agente en ciertas circunstancias. De hecho, una de las motivaciones fundamentales de Barwise y Perry (1983) era dar cuenta de lo que denominan eficiencia del lenguaje: el hecho de que una misma expresión puede ser proferida por diferentes agentes en diferentes ocasiones significando cosas diferentes. Como hemos señalado antes, quien esté familiarizado con los límites clásicos entre semántica y pragmática observará que la semántica de situaciones sobrepasa tales límites,

Aunque existen trabajos que aplican la semántica de situaciones al estudio del significado de otros tipos de actos de habla (como, por ejemplo, las preguntas (Ginzburg (1996)), esta semántica se ha centrado casi exclusivamente en el significado de (proferencias de) oraciones declarativas, tal y como es habitual en las semánticas lógicas más tradicionales.

${ }^{2}$ Es posible pensar que las situaciones podrían definirse por el conjunto de infones que soportan, y esto es útil para muchas cosas. Pero existen importantes razones (véase, por ejemplo, Barwise (1989) o Devlin (1991)) para seguir considerando a las situaciones como primitivos. 
El punto de partida es que las proferencias de oraciones son situaciones en las que hay una hablante, un interlocutor y ciertas circunstancias, que afectan al significado de la proferencia. Como otros tipos de situaciones, las proferencias llevan información sobre otras situaciones más o menos diferentes y remotas respecto a ellas. Veamos algo más detenidamente qué es lo que interviene en tales situaciones.

En primer lugar, hay que considerar la situación de proferencia: la situación o contexto en que se realiza la proferencia. Consideremos un ejemplo. Supongamos que Begoña le dice a Kepa: "Isabel está en la puerta". La situación de proferencia $u$ es el entorno o contexto inmediato en que Begoña profiere la oración y Kepa la escucha. Incluye a ambos Begoña y Kepa, la duración de la proferencia, y contendrá todo lo necesario para identificar los objetos tales como la puerta a la que Begoña hace referencia. Así,

(3) $\quad u \vDash \ll$ Proferir, Begoña, ISABEL ESTÁ EN LA PUERTA, $l, 1 »$ Referir, Begoña, LA PUERTA, D, $l, 1 »$

donde $\mathrm{D}$ es una puerta fijada por $u$.

En muchos casos la proferencia es parte de una situación de discurso más amplia. En cualquier caso la situación de discurso es parte de una situación aun más amplia llamada situación de inmersión. Se dice que la situación de inmersión contiene la parte del mundo directamente relevante para el discurso. Supongamos que Kepa ha pedido antes a Begoña que abra la puerta. Si Begoña responde a tal petición abriendo la puerta habrá ocurrido un cambio en la situación de inmersión. Si e denota la situación de inmersión en el tiempo de la proferencia de Kepa y e' la situación de inmersión un poco más tarde, tendríamos lo siguiente:

$$
\begin{aligned}
& e l=\text { «Cerrada, } \mathrm{D}, l, 1 \text { » } \\
& e^{\prime} \mid=\text { «Abrir, Begoña, } \mathrm{D}, l, 1 \text { » }
\end{aligned}
$$

Finalmente tenemos la situación descrita: la parte del mundo sobre la que versa la proferencia. Ciertos rasgos de la proferencia ayudan a determinar cuál es la situación descrita $s$, por ejemplo:

(5) $\quad s \mid=\ll$ Presente, Isabel, $l, 1 »$

donde $l$ representa la localización de la puerta en el tiempo.

En el marco de la teoría de situaciones se asume que el objetivo principal de la proferencia de oraciones declarativas es proporcionar información. Decíamos antes que cierta relación legaliforme entre el número de anillos de un tronco cortado y la edad de un árbol proporciona información acerca de la edad del árbol en cuestión. Tenemos la misma idea para las situaciones de proferencia. Una relación legaliforme (convencional) entre tipos de situaciones de proferencia y tipos de situaciones descritas es lo que se considera significado en LN.

La oración $\Phi$ : Isabel está en la puerta puede ser proferida por diferentes personas para proporcionar diferente información (con diferentes Isabeles, diferentes puertas, en diferentes tiempos) a diferentes personas. Sin embargo, se supone que permanece una relación abstracta entre el tipo de proferencias de esa oración y el tipo situaciones descritas. El significado de $\Phi$ se define como el vínculo abstracto $\|\Phi\|$ entre dos tipos de situaciones $U$ y $S$, tal que 
(6) $\mathrm{U}=[u|u|=\{$ Hablar-a, $a, b, l, 1 », \ll \operatorname{Proferir}, a, \Phi, l, 1$, », «Referir, $a$, LA PUERTA, $d, l, 1 »\}]$

donde los parámetros $a, b$ y $c$ son para personas y $d$ para $_{\text {puertas }}^{3}$, y

(7) $\mathrm{S}=[s|s|=\ll$ Presente, $c, d, l, 1 »]$.

Así, si tenemos una situación de proferencia en la que Begoña dice $\Phi$ a Kepa, entonces si $u$ es de tipo $\mathrm{U}$, hay una persona, a saber, Begoña, que es asignada al parámetro $a$ correspondiente a la hablante. Begoña profiere $\Phi$ y, en particular, profiere la palabra 'Isabel' para referirse a una persona que es asignada al parámetro $c$, y la expresión 'la puerta' refiriéndose a una determinada puerta que se asigna al parámetro $d$. De este modo, podemos pensar que Begoña tiene la información de que Isabel está en la puerta y que profiriendo $\Phi$ proporciona tal información a Kepa. Este, al escuchar la proferencia, adquirirá la información de que en la situación $s$ hay una persona determinada llamada Isabel que está en la puerta; en otras palabras, adquirirá la información de que la situación $s$ es de tipo S. A esta información se le denomina contenido proposicional de la proferencia, que consiste en

(8) $s \mid=\ll$ Presente, Isabel, $l, 1 »$

Esta es la información que la proferencia proporciona sobre la situación descrita y lo es en virtud del significado de la oración $\Phi,\|\Phi\|$, que proporciona el vínculo entre la situación de proferencia y la situación descrita, y hace posible que una proferencia de $\Phi$ sea informativa.

\section{La teoría de representación del discurso o DRT}

Uno de los principales objetivos de DRT es, como su nombre indica, el relacionado con el estudio del discurso y no únicamente de las oraciones aisladas de todo contexto como era el caso de la gramática de Montague. Otra de sus características principales tiene que ver con la representación. DRT propone un nivel intermedio de representación semántica situado entre las expresiones lingüísticas y los modelos de la realidad, nivel donde se almacena la información dada por el discurso. En DRT el proceso que va desde las expresiones a las representaciones no es composicional y esto hace que el nivel de representación sea esencial.

Una tercera característica de DRT es que es considerada como un puente entre dos perspectivas que, tradicionalmente, se han considerado en semántica como opuestas: la perspectiva psico-lingüística que relaciona estructuras sintácticas con representaciones mentales y la perspectiva lógica que relaciona estructuras sintácticas con (modelos de) la realidad. Dicho de otra forma, DRT trata de ligar el punto de vista declarativo o estático del significado con el punto de vista procedural o dinámico.

Algunas de las motivaciones empíricas primeras de DRT vienen principalmente del estudio de la interpretación de pronombres y relaciones anafóricas entre pronombres y términos indefinidos.

\subsection{Descripción informal de $D R T$}

En DRT cada oración tiene asignada una representación que se consigue mediante la conversión de las estructuras sintácticas en las llamadas "estructuras de representación del

${ }^{3}$ En la presente exposición podemos interpretar los parámetros como variables individuales. 
discurso" (DRSs, "Discourse Representation Structures"). Expliquemos mediante un ejemplo el proceso de creación de una DRS. Para representar oraciones simples tales como

(9) Mary kicked a donkey.

necesitamos introducir un referente del discurso para el nombre propio Mary. Así obtenemos:

\begin{tabular}{|l|}
\hline $\mathrm{x}$ \\
\hline Mary(x) \\
$\mathrm{x}$ kicked a donkey \\
\hline
\end{tabular}

También el término indefinido "a donkey" introduce un referente de discurso. Así que introducimos una nueva entidad "y" para substituir en la oración "a donkey" de la siguiente forma:

\begin{tabular}{|l|}
\hline $\mathrm{x} \mathrm{y}$ \\
\hline Mary $(\mathrm{x})$ \\
donkey(y) \\
$\mathrm{x}$ kicked $\mathrm{y}$ \\
\hline
\end{tabular}

Si la anterior oración estuviera contenida en un discurso tal como:

(10) Mary kicked a donkey. It kicked her too.

podríamos añadir más información a la DRS anterior proveniente de la segunda oración de la siguiente forma:

\begin{tabular}{|l|}
\hline $\mathrm{x}$ y u v \\
\hline Mary(x) \\
donkey(y) \\
x kicked y \\
$\mathrm{u}=\mathrm{y}$ \\
$\mathrm{v}=\mathrm{x}$ \\
$\mathrm{u}$ kicked $\mathrm{v}$ \\
\hline
\end{tabular}

donde tenemos un conjunto de referentes de discurso $(\{\mathrm{x}, \mathrm{y}, \mathrm{u}, \mathrm{v}\})$ y un conjunto de fórmulas llamadas condiciones (Mary(x), donkey(y), x kicked $\mathrm{y}, \ldots)$ 
Las DRSs se consideran modelos parciales de la realidad, así por ejemplo en la DRS de arriba el modelo parcial tendría dos individuos que verificarían las propiedades dadas por las condiciones. Diremos que una DRS es verdadera en un modelo M (que se define usualmente como en lógica de primer orden) si el modelo parcial asociado a la DRS se puede sumergir en M. Definimos de esta forma la idea de inmersión de una DRS en un modelo M como una función f que asigna elementos del dominio de $\mathrm{M}$ a los referentes de discurso de la DRS de tal forma que todas y cada una de las condiciones en la DRS sean verdaderas en M para estos elementos. Así la DRS anterior será verdadera si y sólo si existe una inmersión verificadora $\mathrm{f}$ que asigna individuos a los referentes $x, y, u, v$ de forma que $f(x)=M a r y, f(y)=$ donkey, $f(x)=f(v), f(y)=f(u)$ y tales que $f(x)$ kicked $f(y), f(u)$ kicked $f(v)$.

En toda oración hay incluida una referencia al tiempo en el cual tuvo lugar la acción con relación al momento de la proferencia. Para expresar las referencias temporales DRT utiliza referentes de discurso especiales que representan estados, $\mathrm{si}_{\mathrm{i}}$ (para el caso de verbos estáticos), eventos, $\mathrm{e}_{\mathrm{i}}$, (para verbos no estáticos), tiempo, $\mathrm{t}_{\mathrm{i}}$, considerado normalmente como intervalos continuos. Un elemento distinguido es el referente de discurso $n$, que representa el momento de la proferencia. Además entre estos referentes de discurso puede haber determinadas relaciones entre las que resaltan precedencia, representada por < en el caso de precedencia estricta, inclusión temporal, $\subseteq$, y simultaneidad representada por $\sim$.

Así si queremos representar el discurso en (10) teniendo en cuenta las referencias temporales, tendríamos la siguiente DRS:

\begin{tabular}{|l|}
\hline $\mathrm{x} \mathrm{y} \mathrm{u} \mathrm{v} \mathrm{e}_{1} \mathrm{e}_{2} \mathrm{n}$ \\
\hline Mary(x) \\
donkey(y) \\
$\mathrm{e}_{1}-\mathrm{x}$ kicked $\mathrm{y}$ \\
$\mathrm{e}_{1}<\mathrm{n}$ \\
$\mathrm{u}=\mathrm{y}$ \\
$\mathrm{v}=\mathrm{x}$ \\
$\mathrm{e}_{2}-\mathrm{u}$ kicked $\mathrm{v}$ \\
$\mathrm{e}_{2}<\mathrm{n}$ \\
$\mathrm{e}_{1}<\mathrm{e}_{2}$ \\
\hline
\end{tabular}

Sea ahora la oración siguiente:

(11) At 7:30 Mary was running when a donkey kicked her.

Su DRS asociada sería: 


\begin{tabular}{|l|}
\hline$x$ y u $s_{1} e_{2} \mathrm{nt}_{1}$ \\
\hline Mary $(x)$ \\
$7: 30\left(t_{1}\right)$ \\
$s_{1}-$ x run \\
$s_{1}<n$ \\
$t_{1} \sim s_{1}$ \\
donkey(y) \\
x=u \\
$e_{2}-$ y kicked u \\
$e_{2}<n$ \\
$e_{2}<s_{1}$ \\
$e_{2} \sim t_{1}$ \\
\hline
\end{tabular}

\subsection{DRSs complejas}

Hay oraciones que contienen conceptos lógicos como la condicionalidad, la cuantificación universal o la negación que no pueden ser representadas por medio de las DRSs simples que hemos presentado hasta ahora. A modo de ejemplo indicaremos cómo se representaría la oracion:

(12) If Mary owns a donkey then she beats it.

\begin{tabular}{|l|l|}
\hline \multicolumn{1}{|l|}{$\mathrm{M}$} \\
\hline $\mathrm{y}$ \\
\hline \\
\hline \\
\hline
\end{tabular}


Las condiciones de verdad para DRSs de la forma $\mathrm{K}_{1} \rightarrow \mathrm{K}_{2}$ donde $\mathrm{K}_{2}$ es una DRS que expande a $\mathrm{K}_{1}$ serían ahora las siguientes: $\mathrm{K}_{1} \rightarrow \mathrm{K}_{2}$ se satisface en un modelo $\mathrm{M}$ si toda situación en $M$ que satisface a $K_{1}$ satisface también a $K_{2}$. Más formalmente, $K_{1} \rightarrow K_{2}$ es verdadera en $\mathrm{M}$ con respecto a una inmersión f si y sólo si siempre que se pueda extender $\mathrm{f}$ a una inmersión g para la que $\mathrm{K}_{1}$ es verdadera en $\mathrm{M}$, ésta se puede extender también a una inmersión h para la que $\mathrm{K}_{2}$ es verdadera en $\mathrm{M}$.

En el caso de la representación de la negación necesitamos introducir también un nuevo tipo de DRSs complejas que veremos con más detalle en el siguiente apartado.

\section{LA NEGACIÓN}

La semántica de la negación es uno de esos problemas que, planteado por primera vez en Aristóteles, no ha dejado nunca de generar gran debate a lo largo de toda la historia de la filosofía, la filosofía del lenguaje y la lingüística. No pretendemos reproducir aquí ese debate (véase Horn (1989)), ni abordar toda la problemática de aspectos ontológicos, psicológicos, sintácticos semánticos y pragmáticos ligada la negación. Trataremos el problema de su interpretación en el tipo de semánticas aquí expuestas.

Jackendoff (1972) introduce el siguiente ejemplo:

(13) Max didn't kill the judge with a hammer.

Fuera de contexto la mayoría de los oyentes interpretarían esta oración de la siguiente manera: hay alguien llamado Max y hay un juez pero ese Max no usó un martillo para matar a ese juez.

El hecho de que en una interpretación de este tipo, que podemos considerar la más neutra en cuanto que no exige un contexto especial, se suponga que hay alguien llamado Max se interpreta como que el sujeto queda fuera del operador de negación y se conoce en la literatura como el problema de la fuerza existencial de los sujetos en oraciones negativas. El hecho de que se suponga que Max mató al juez se interpreta como que el operador de negación, de hecho, no niega todo el SV sino sólo una parte de él. Estos problemas, relacionados con la posibilidad de escapar del alcance de la negación de algunos elementos de la oración estaban ya planteados en Aristóteles.

Frege (1882), Strawson (1950) o Kamp (1981), entre otros, se han referido al asunto de que la mayoría de los sujetos de aserciones negativas tienen fuerza existencial afirmando que esto les hace quedar fuera del operador de negación. Russell (1905) argumentó que el sujeto debe analizarse como un cuantificador existencial y que se producen interacciones de alcance lógico entre la negación y este existencial dando lugar a una lectura donde el sujeto tiene fuerza existencial (alcance corto de la negación) y a otra donde no se afirma su existencia (alcance amplio). En Jackendoff (1972), por otro lado, se sostiene que algunas partes del predicado de oraciones negativas, dependiendo del contexto, parecen no quedar afectadas por este operador y, por tanto, que deberían quedar fuera de su alcance.

Horn (1989), donde se recogen estos y otros muchos problemas y especulaciones acerca de la negación natural, adopta otra perspectiva. Mantiene que la negación debe ser considerada a nivel semántico un operador externo a la oración y que tanto el problema de la fuerza 
existencial de los sujetos como el asunto de qué partes del predicado pueden quedar sin negar debe ser explicado a otro nivel, no-veritativo-condicional. Siguiendo a Strawson (1964) y a Reinhart (1981) afirma que los sujetos tienden a ser temas y los temas quedan fuera de lo que se afirma pragmáticamente y, de la misma manera, fuera de lo que se niega pragmáticamente.

Vallduví (1990), partiendo de los resultados de Horn, comienza a desarrollar la idea, implícita o explícita en muchas propuestas anteriores, de que estos efectos de pérdida de alcance del operador de negación son debidos al componente informacional, a la división de las oraciones en partes más informativas (el foco o elementos focales) y partes que sirven para anclar esa información (temas o elementos temáticos). Pero al considerar sólamente la semántica de cambio de archivo de Heim no puede formalizar adecuadamente sus resultados. Hace falta un marco formal donde pueda representarse tanto el significado semántico de la oración como el contenido pragmático necesario para construir las distintas interpretaciones de aserciones negativas.

En este trabajo deseamos limitarnos a dos problemas:

1. Exponer varias formas de representar la negación en SS y DRT, valorando si son suficientes para modelar la negación del lenguaje natural.

2. Introducir una nueva teoría de la partición informacional en el marco de una extensión de DRT, SDRT, que puede mostrarse adecuada para estudiar la formación del alcance de la negación en oraciones que pertenecen a un discurso y su interacción con las nociones de foco y de tema.

Pretendemos mostrar que la representación de la negación en una teoría semántica no es un asunto trivial. Habitualmente se presentan diferentes posibilidades con diferentes consecuencias de orden lingüístico, psicológico e incluso ontológico que suelen afectar de manera importante a las bases conceptuales de tales teorías.

Tal y como se ha reconocido en anteriores estudios sobre la negación, la noción de foco, relacionada en inglés con el acento más prominente de la proferencia, puede servir para fijar el alcance del negador. En la oración de Jackendoff, si situamos el acento principal en the judge (marcado mediante mayúsculas):

(14) Max did not kill THE JUDGE with a hammer.

La primera lectura que hace cualquier angloparlante es que Max, al parecer, mató a alguien con un martillo, pero ese alguien no es el juez. La negación parece coger como alcance sólo the judge. Esta interpretación es más restringida que la interpretación que considera que el alcance de la negación es toda la oración o todo el sintagma verbal y ha sido denominada negación interna (frente a la otra negación, denominada externa).

Pero tenemos además otras lecturas que surgen de situar el acento principal de la oración en distintos sitios:

(15) Max did not KILL the judge with a hammer.

Max did not kill the judge with a HAMMER.

MAX did not kill the judge with a hammer. 
Se ha dicho que el acento principal indica foco informacional y que este foco se constituye en alcance de la negación interna. Vamos a ver algunas formas posibles de modelar la negación en SS y DRT y valorar hasta qué punto resultan adecuados.

\subsection{La negación en Semántica de Situaciones}

No existe un tratamiento definitivo de la negación en semántica de situaciones, y disponemos de varias alternativas para tratar de resolver lo que se ha denominado el problema de la asimetría de las oraciones negativas (Devlin (1991)).

Es habitual suponer que (una proferencia de) la oración afirmativa siguiente:

(16) Max killed the judge with a hammer.

describe un estado de cosas. En SS tendríamos una situación descrita $s$, tal que $s \mid=\ll \mathrm{KILL}, \mathrm{M}$, $\mathrm{J}, \mathrm{H}, l, 1 »$, donde $\mathrm{M}, \mathrm{J}$ y $\mathrm{H}$ son los objetos denotados por Max, the judge y a hammer respectivamente (estamos considerando que a hammer es referencial para evitar mezclar problemas), y $l$ la ubicación espacio-temporal.

Por otro lado, para modelar la proferencia de una instancia de la oración negativa:

(17) Max did not kill the judge with a hammer.

tenemos que decidir (al menos) dos cuestiones importantes:

1. Considerar que los infones negativos están soportados por una situación total, un mundo; o, por el contrario, considerar que también las situaciones parciales soportan infones negativos. A favor de la segunda opción tenemos que se conserva la parcialidad (señal de identidad de ST y de la interpretación de muchos fenómenos de LN para esta teoría).

2. No permitir infones negativos en la teoría considerando que los problemas de tipo ontológico ${ }^{4}$ - ¿existen los hechos negativos?- y de tipo epistemológico -¿Cómo percibimos tales hechos negativos?- son suficientes para aconsejar su eliminación. Utilizar sólo la relación de no-soporta, $\mid \neq$, para expresar una oración negativa como que no existe ninguna situación que soporte su contrapartida positiva. O, la alternativa de permitir infones negativos.

Si adoptamos la perspectiva de utilizar mundos totales y de permitir infones negativos obtenemos la representación:

(18) $\quad w \mid=\ll \mathrm{KILL}, \mathrm{M}, \mathrm{J}, \mathrm{H}, l, 0 »$

\footnotetext{
${ }^{4}$ Que la ontología del LN y la ontología real no son isomórficas ha sido mostrado, por lo menos, con la observación de que necesitamos individualizar eventos y estados entre los objetos abstractos de la ontología del LN y, sin embargo, no tenemos tal distinción entre objetos del mundo (es decir, eventos y estados refieren a los mismos objetos del mundo). Esto significa que los supuestos problemas ontológicos no pueden tomarse como decisivos a la hora de decidir si utilizar o no infones negativos en la teoría. Es decir, en el mundo puede que no haya hechos negativos pero eso no impide adoptar una ontología para el LN donde se permitan infones negativos soportados por situaciones si consideramos su inclusión necesaria para explicar adecuadamente fenómenos del LN.
} 
Si adoptamos la perspectiva de utilizar mundos totales y no permitir infones negativos, tenemos:

$$
w \mid \neq \ll \mathbb{K I L L}, \mathrm{M}, \mathrm{J}, \mathrm{H}, l, 1 »
$$

Mientras que (20) parece que puede llevarnos (desde una postura filosófica realista extrema) a asumir la existencia de hechos negativos, la segunda opción parece que no acarrearía este tipo de problemas.

Pero ocurre que, puesto que hemos considerado que la situación descrita es el mundo $w$, no sólo se cumple que

$$
\text { si } w \mid=« \mathrm{KILL}, \mathrm{M}, \mathrm{J}, \mathrm{H}, l, 0 \text { » entonces } w \mid \neq « \mathrm{KILL}, \mathrm{M}, \mathrm{J}, \mathrm{H}, l, 1 »,
$$

sino que, dado que $w$ es una situación total, también,

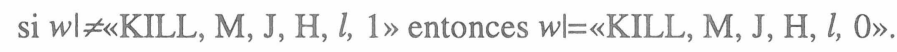

Es decir, si adoptamos w como la parte del mundo apropiada para soportar representaciones de oraciones negativas, la decisión de permitir o no infones negativos es trivial ya que tenemos que son equivalentes.

Supongamos ahora que las (proferencias de) oraciones negativas pueden describir situaciones parciales. En nuestro ejemplo parece natural suponer que todas las lecturas pueden describir una situación parcial que soportaría el contenido proposicional siguiente:

$$
s \mid \neq \ll \mathrm{KILL}, \mathrm{M}, \mathrm{J}, \mathrm{H}, l, 1 »
$$

Este sería el modo de representar la negación que hemos denominado aristotélica, una negación externa, que sería más débil que las lecturas donde se especifican alcances parciales del negador. También tenemos la posibilidad, si aceptamos infones negativos, de modelar el contenido de la proferencia de la siguiente manera:

$$
\mathrm{s} \mathrm{l}=\ll \mathrm{KILL}, \mathrm{M}, \mathrm{J}, \mathrm{H}, l, 0 »
$$

Los "hiperrealistas" preferirán el primer camino, mientras que los conceptualistas moderados optarán por el segundo. No nos vamos a detener en los pros y contras de cada opción (entre las/os autoras/es se dan ambas posturas) y sólo vamos a señalar lo qué ocurre cuando, tomando la segunda opción, la situación soporta más información acerca de la misma proferencia. Veamos qué otros infones puede soportar s:

$$
\begin{aligned}
& \mathrm{s}_{1}=\sigma \wedge<<\text { killer, M, s; } 1 \gg>\wedge<<\text { killed, J, s; 0 }>\wedge \wedge<<\text { mood, H, s; 0 > } \\
& \mathrm{s}_{2} \mid=\sigma \wedge<<\text { killer, M, s; 1 }>\wedge \wedge<<\text { killed, J, s; 1 }>\wedge \wedge<<\text { mood, H, s; 0 > } \\
& \mathrm{s}_{3} \mid=\sigma \wedge<<\text { killer, M, s; 1 }>>\wedge<<\text { killed, J, s; 1 }>\wedge \wedge<<\text { mood, H, s; 1 > } \\
& \mathrm{s}_{4} \mid=\sigma \wedge<<\text { killer, M, s; 0 }>\wedge<<\text { killed, J, s; 0 }>\wedge \wedge<<\text { mood, H, s; 0 > } \\
& \mathrm{s}_{5} \mid=\sigma \wedge<<\text { killer, M, s; 0 }>\wedge<<\text { killed, J, s; 0 }>\wedge \wedge<<\operatorname{mood}, \mathrm{H}, \mathrm{s} ; 1>> \\
& \mathrm{s}_{6} \mathrm{l}=\sigma \wedge<<\text { killer, M, s; 0 }>\wedge<<\text { killed, J, s; } 1 \gg>\wedge<<\operatorname{mood}, \mathrm{H}, \mathrm{s} ; 1 \gg>
\end{aligned}
$$

Es evidente que estas situaciones no pueden ser la misma ya que soportan diferentes conjuntos de infones. Ahora bien, todas tienen en común que soportan el infón $\sigma$. Es decir, 
todas tienen como parte una situación s que soporta exclusivamente $\sigma$. Esto puede significar que tomando esta opción podríamos modelar tanto la negación externa e interna del LN utilizando un fragmento de ST con situaciones parciales y guardando la persistencia (es decir, si una situación apoya un infón y es parte de otra situación, entonces esta situación también apoyará ese infón). Pero obsérvese que estos resultados probablemente no podrán mantenerse si consideramos un fragmento de LN que además de negación incluya cuantificadores.

Concluimos esta sección señalando que hemos mostrado varias formas posibles de modelar la negación en ST y hemos visto que al menos en una de ellas seguimos conservando parcialidad y persistencia, características básicas del marco situado. Esto significa que es un marco adecuado para representar la negación natural. Ahora bien, dado que no tiene integrada una teoría de la partición informacional, podemos concluir que no tiene mecanismos para determinar el alcance de la negación interna en proferencias concretas. Veremos que éste será asimismo el resultado principal con respecto a DRT en la próxima sección.

\subsection{La negación en DRT}

DRT introduce una subDRS bajo el alcance del operador lógico de negación para dar cuenta de la negación natural. Así la negación de todo el SV de nuestro ejemplo adopta la forma siguiente:

(20) $\quad \operatorname{Max} \neg$ (killed the judge with a hammer)

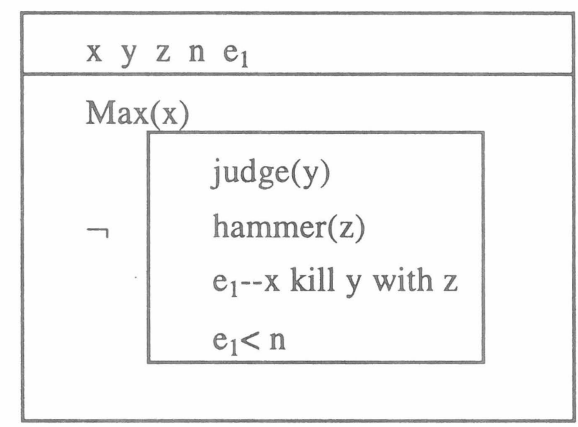

Las condiciones de verdad para una DRS de la forma $\neg \mathrm{K}$ son las siguientes: $\neg \mathrm{K}$ se satisface en un modelo $\mathrm{M}$ para una inmersión $\mathrm{f}$ ssi no se puede combinar $\mathrm{f}$ con una inmersión g que asocia objetos del dominio de $\mathrm{M}$ con los referentes del discurso de $\mathrm{K}$ de tal forma que la combinación de f y g sea una inmersión que satisfaga a la DRS en M.

Si tenemos en cuenta las observaciones hechas anteriormente, y consideramos que no siempre es ésta la representación de las condiciones de verdad de una proferencia de (19) y quisieramos representar la proferencia en la que enfatizamos "the judge": 


\begin{tabular}{|l|}
\hline $\mathrm{x} \mathrm{y} \mathrm{z} \mathrm{n} \mathrm{e}$ \\
\hline $\operatorname{Max}(\mathrm{x})$ \\
$\neg \quad$ judge(y) \\
Hammer(z) \\
$\mathrm{e}_{1-\mathrm{x}}$ kill y with $\mathrm{z}$ \\
$\mathrm{e}_{1}<\mathrm{n}$ \\
\hline
\end{tabular}

De una forma análoga podríamos representar el resto de los alcances y dar las condiciones de verdad apropiadas. Eso significa que DRT es igualmente adecuada como marco semántico para tratar la negación. Pero necesita incorporar una teoría de la partición informacional para poder dar cuenta del proceso de formación del alcance de la negación y de la interacción de este alcance con el foco. Utilizaremos una extensión de DRT, denominada DRT con Segmentos o SDRT, que tiene integrada una teoría de la partición informacional para ver cómo se determina esa interacción entre negación y estructura informacional.

\section{LA NEGACIÓN EN SDRT}

Comenzaremos presentando algunos de los supuestos generales que deben configurar una teoría de la partición informacional integrada en una teoría del discurso (Asher y Gómez Txurruka (1996)):

1. La partición informacional (PI) de una proferencia es un fenómeno que ocurre en el nivel del discurso (y no a nivel intraoracional), y que tiene que ver, en último término, con la forma en que la hablante y el oyente generan/procesan el LN. La partición informacional contribuye a la eficiencia en el procesamiento y a la coherencia en el discurso.

2. Todas las proferencias tienen partición informacional.

3. Dada una oración, la sintaxis y la entonación (en inglés) proporcionan un conjunto de Particiones Informacionales Posibles (PIPs).

4. Hay dos categorías informacionales: tema (entidad sobre la que versa la oración) y foco (información específica que completa o corrige parte de una situación dada).

5. En base a los primitivos de foco y tema tenemos (al menos) dos tipos de PI: estructuras de dominancia focal (construidas alrededor de un foco) y de dominancia temática (construidas alrededor de un tema).

6. En una gramática de estructura sintagmática, el elemento dominante de una PI de una proferencia es siempre sintácticamente construible, esto es, es un constituyente de la gramática. 
7. Hay una fuerte interacción entre las Relaciones de Discurso y la Partición Informacional. Un tipo de interacción se concreta en que cada Relación de Discurso exige una Partición Informacional determinada.

8. La Partición Informacional juega un papel crucial en la determinación del contenido de oraciones que incorporan adverbios cuantificacionales (only, even, too, also, always, etc.), genéricos, etc. También el operador de negación de LN utiliza la Partición Informacional para determinar su alcance en la oración.

Dado el tipo de teoría de la partición informacional que asumimos necesitamos una teoría del discurso que venga provista de una teoría de las relaciones de discurso. SDRT es precisamente una teoría de tales características.

\subsection{Introducción a SDRT}

SDRT (Asher 1993) extiende la semántica dinámica de DRT de forma no trivial. Para esta teoría un discurso no produce simplemente una DRS sino una estructura compleja donde las DRSs están relacionadas mediante relaciones de discurso dando lugar a Estructuras Segmentadas de Representación del Discurso o SDRSs. También las SDRSs pueden relacionarse entre sí mediante relaciones de discurso produciendo estructuras jerárquicas de representación. Así, una SDRS consiste en un conjunto de DRSs y SDRSs y un conjunto de relaciones de discurso que se predican de esos constituyentes. Supongamos que hemos construido la SDRS $\tau$ de las $n-1$ primeras oraciones de un discurso. Dada la oración $a_{n}$, el proceso de construcción tiene lugar en cuatro fases:

(i) Construcción de la DRS para la oración $a_{n}$.

(ii) Especificación de los sitios de atadura para la nueva DRS.

(iii) Deducción de la RD entre la nueva DRS y el sitio de atadura.

(iv) Aplicación de principios de revisión, si procede.

En la fase (i) procedemos a construir la DRS para la nueva oración siguiendo el algoritmo de DRT. Las fases (ii) y (iii) determinan los sitios que se hayan libres en el modelo del discurso previo para poder atar ahí la DRS de la nueva oración y, asímismo, el tipo de relación de discurso que se utilizará para poder obtener un modelo unificado. La determinación de la relación de discurso tiene en cuenta numerosas fuentes de información (sintáctica, semántica, pragmática, léxica, etc.) y se utilizará una lógica no-monótona (con una noción de inferencia monótona y otra no-monótona) para explicar cómo estas distintas fuentes contribuyen a la determinación de la relación de discurso adecuada en un contexto. La última fase recoge la necesidad de actualizar el modelo de discurso de acuerdo a retricciones desencadenadas por las relaciones de discurso. Dadas las limitaciones de espacio, no podemos extendernos en la descripción de estos estadios y referimos al/a lector/a interesado/a a Asher (1993).

\subsection{Interpretación de la negación en SDRT}

Necesitamos, asimismo, postular una hipótesis de estructura informacional para el operador de negación y su alcance. Vamos a mantener que not puede pertenecer a los elementos focales o temáticos de la proferencia. Si es focal entonces el alcance se construye en la propia proferencia y estará formado por el resto de condiciones en foco. Si es temático 
entonces el alcance será heredado. Puede haber herencia directa: si la negación aparece en la proferencia ancla, determina en ella su alcance y es apropiado para ser heredado. Puede haber herencia indirecta: si en el ancla no aparece una negación cuyo alcance pueda heredarse; tendremos que recurrir información inferida del ancla para poder determinar el alcance (pueden ser creencias, intenciones, implicaturas, etc. inferidas del ancla las que proporcionen el alcance al negador).

Esta hipótesis es una primera aproximación y requiere más refinamiento y contrastación. Pero, aunque sea provisional, puede servir para imponer una importante restricción informacional sobre la negación en el discurso.

Vamos a proceder seguidamente a la construcción de un modelo para un mini-discurso donde el alcance de la negación se construye en la misma proferencia en interacción con el contexto (i.e., no se hereda).

(21) (Supongamos que estamos en un juicio donde se juzga a Max por varios asesinatos)

Testigo: Max killed the judge with a HAMMER.

Fiscal: No, Max did not kill THE JUDGE with a hammer.

He killed the judge's DOG in this way.

La partícula no que antecede a la primera proferencia del fiscal indica (por defecto) que estamos ante una corrección. La relación de discurso de Corrección está restringida de forma crucial por la partición informacional (Asher y Gómez Txurruka (1995)). Informalmente, tenemos una restricción que nos dice que para que haya Corrección tenemos que correlacionar (i) el foco de la proferencia correctora con alguna parte de la proferencia ancla y (ii) los elementos temáticos de la proferencia correctora con el resto del ancla de tal manera que el conjunto de (ii) constituye una situación consistente y si le añadimos la información del foco y su imagen obtenemos inconsistencia.

Comenzamos construyendo las DRSs de las proferencias:

$\mathrm{T}: \alpha$
\begin{tabular}{|l|}
$\mathrm{x}, \mathrm{y}, \mathrm{z}, \mathrm{e}, \mathrm{t}$ \\
\hline $\mathrm{e}-\mathrm{kill}(\mathrm{x}, \mathrm{y})$ \\
$\operatorname{Max}(\mathrm{x})$ \\
the judge(y) \\
a hammer(modo(e): $\mathrm{z})$ \\
t<ahora \\
\hline
\end{tabular}




\begin{tabular}{|c|}
\hline$x^{\prime}, y^{\prime}, z^{\prime}, e^{\prime}, t^{\prime}$ \\
\hline$e^{\prime}-\operatorname{kill}\left(x^{\prime}, y^{\prime}\right)$ \\
\hline $\operatorname{Max}\left(x^{\prime}\right)$ \\
\hline the judge(y') \\
\hline a hammer(modo(e'): $\left.z^{\prime}\right)$ \\
\hline t'<ahora \\
\hline$\neg \mathrm{a}$ \\
\hline$a=$ ? \\
\hline
\end{tabular}

La negación introduce dos condiciones en la DRS: $\neg$ a y a=?. $\neg$ a indica que la negación tiene como alcance $a . a=$ ? indica que a es una anáfora que tenemos que resolver pragmáticamente. Seguidamente consideramos el conjunto de particiones informacionales posibles de la primera oración del fiscal.

Conjunto de PIPs=

$\left\{\beta_{1}:\right.$ Max did /not/ kill /the JUDGE/ with a hammer.

$\beta_{2}$ : Max did /not kill the JUDGE/ with a hammer.

$\beta_{3}$ : Max did not/kill the JUDGE/ with a hammer.

$\beta_{4}$ : Max did not kill /the JUDGE/ with a hammer.\}

¿Cómo elegimos la PIP adecuada en este contexto?. Tenemos como hipótesis (por defecto) que hay Corrección. Para que el conjunto de elementos temáticos y su imagen sea consistente el negador tiene que estar en foco (sino tendríamos kill y do not kill en este conjunto). Esto elimina $\beta_{3}$ y $\beta_{4}$.

Las otras dos PIPs tienen not en posición focal El resto del material en foco será precisamente el alcance del negador. La única pista que tiene el oyente para seleccionar una de estas dos PIPs viene dada por la existencia de una tendencia a seleccionar el foco más corto (coincidente con el foco entonacional) en ausencia de información contraria. Por tanto, el oyente seleccionará $\beta_{1}$ y esta elección se verá confirmada tras el procesamiento de la segunda proferencia del fiscal: He killed the judge's DOG in this way. La partición informacional de esta oración en este contexto sería: He killed /the judge's DOG/ in this way, que nos permite concluir que la estructura temática (heredada) de $\beta$ es de la forma:

Max killed $x$ with the hammer

Por tanto, hemos visto que usando la restricción informacional sobre Correcciones y la preferencia por parte de los interpretadores a usar el foco corto en las estructuras de dominancia focal, obtenemos la siguiente PI:

Max did /not/ kill /the JUDGE/ with a hammer. 
Y esta selección se verá confirmada al procesar la siguiente proferencia. La selección de la partición informacional permite resolver la anáfora $\mathrm{a}=$ ?, dado que al estar not en posición focal, el resto de elementos focales constituye su alcance. Obtenemos la siguiente DRS resuelta:

\begin{tabular}{|c|c|}
\hline $\mathrm{x}$ & $y^{\prime}, z^{\prime}, e^{\prime}, t^{\prime}$ \\
\hline \multirow{5}{*}{$\neg$} & $e^{\prime}-\operatorname{kill}\left(x^{\prime}, y^{\prime}\right)$ \\
\hline & the judge(y') \\
\hline & a hammer(modo(e'): $\left.z^{\prime}\right)$ \\
\hline & $\operatorname{Max}\left(x^{\prime}\right)$ \\
\hline & $\mathrm{t}^{\prime}<$ ahora \\
\hline
\end{tabular}

La discusión de este ejemplo podría llevarse a cabo de manera formal introduciendo axiomas acerca de relaciones de discurso y una lógica no-monótona. Quizá también resultaría interesante introducir un orden en el conjunto de PIPs para fijar algunas de las preferencias.

Puesto que SDRT utiliza la construcción de DRSs de la DRT es obvio que podemos asimismo obtener un modelo de la proferencia donde el negador sea externo. Pero para obtener esa lectura menos informativa no necesitamos utilizar un marco formal de la riqueza expresiva de SDRT. Podemos quedarnos en DRT.

Un comentario final sobre el ejemplo. Conviene señalar que, si $\beta$ no coge ese alcance sino otro distinto, el discurso que obtenemos es incoherente: no podremos concluir que se cumple la relación de Corrección y, consecuentemente, no podremos atar esa proferencia al discurso. Así que el alcance del negador contribuye a la coherencia de los discursos.

\section{CONCLUSIÓN}

En este trabajo hemos dado una caracterización a grandes rasgos de dos de las semánticas formales con mayor éxito en la tarea de dar cuenta del significado del lenguaje natural, la semántica de situaciones y la teoría de representación del discurso. Seguidamente, hemos aplicado ambas semánticas a la interpretación del operador de negación observando que en ellas podemos representar satisfactoriamente tanto la negación externa como interna pero que no sirven para determinar el alcance de la negación interna. Hemos visto que este alcance se determina en interacción con el mecanismo de partición informacional y esto nos obliga a utilizar un marco semántico más rico y flexible que los anteriores, la teoría de representación del discurso con segmentos, que es una extensión sustancial de DRT y tiene integrada una teoría de la partición informacional desde el punto de vista del discurso. 


\section{REFERENCIAS}

Asher, Nicholas (1993): Reference to abstract objects in discourse. Dordrecht: Kluwer Academic Press.

Asher, Nicholas y Isabel Gómez Txurruka (1995): Extending SDRT to integrate a theory of the informational partition. Report No. ILCLI-95-LIC-2, UPV-EHU.

Barwise, Jon (1989): The situation in Logic. Stanford: CSLI.

Barwise, Jon y John Perry (1981): 'Situations and Attitudes', Journal of Philosophy 78, 668-691.

Barwise, Jon y John Perry (1983): Situations and Attitudes. Cambridge, Ma: MIT Press.

Devlin, Keith (1991): Logic and Information, Cambridge: Cambridge University Press.

Frege, Gottlob (1882): "On sense and reference". Translated and reprinted in P. T. Geach y M. Black (eds.), Translations from the philosophical writings of Gottlob Frege. Oxford: Blackwell, 1952. Págs. 56-78.

Ginzburg, Jonathan (1996): "Resolving questions. Part I", Linguistics and Philosophy 18, 459-527.

Horn (1989): A Natural History of Negation. Chicago: University of Chicago Press.

Jackendoff, Ray (1972): Semantic interpretation in generative grammar. Cambridge: MIT Press.

Kamp, Hans (1981): "A theory of truth and semantic interpretation", in J. Groenendijk et al. (eds.), Formal methods in the study of language. Amsterdam: Mathematical Centre Tracts. Págs. 277322.

Kamp, Hans y Uwe Reyle (1994): From discourse to logic. Dordrecht: Kluwer Academic Publishers.

Reinhart, Tanya (1982): "Pragmatics and Linguistics: An analysis of sentence topics", Philosophica 27, 53-94.

Russell, Bertrand (1905): “On denoting”, Mind 14, 479-93.

Strawson, Peter F. (1950): “On referring”, Mind 59, 320-44.

Strawson, Peter F. (1964): "Identifying reference and truth-values". Reprinted in D. Steinberg y L. Jacobovits (eds.), Semantics. Cambridge: cambridge University Press. Págs. 86-99.

Vallduví, Enric (1990): The informational component. University of Pennsylvania: Ph. D. diss. 
